\title{
Supporting a University Satellite Engineering Team via Inclusivity and Initiative
}

\author{
William E Crofts \& Julia Hunter-Anderson \\ School of Engineering \\ University of Warwick \\ Coventry, UK \\ w.e.crofts@warwick.ac.uk J.Hunter-Anderson@warwick.ac.uk
}

\begin{abstract}
Any university-based satellite engineering team that hopes to design, build, launch and operate even the most modest of satellite missions is likely to encounter enormous resourcing difficulties. Unless you are fortunate enough to receive support from, e.g. a well-funded research group, it is normally extremely difficult to raise the amount of funding needed. In a research-led university, such a 'practical' project rarely has the 'currency' of research publication output even if it carries potentially very high profile exposure for the department/university.
\end{abstract}

The University of Warwick Satellite (WUSAT) Engineering Programme has found ways to overcome this ongoing problem ever since its inception in 2006. This paper describes how resourceful and inventive the Directors and students have had to be in order to facilitate the achievements that the team has made over the past thirteen years.

What is described in this paper is not just a mechanistic approach in 'how to get things done on a tight budget', it is also a description of how to develop an attitude and a culture that makes a wide range of individuals and organisations feel that they are part of the wider 'WUSAT Team'. It is an illustration of 'thinking on your feet' when an opportunity arises for you to offer something to someone else rather than just thinking about what you want from them. The reward for the team will come in the payback that almost inevitably comes from organisations/individuals whose trust and respect you have earned.

This paper describes many examples of such relationship-forging events. These include examples involving,

- Partner companies,

- Warwick University staff/resources,

- The inclusivity and diversity of the wider WUSAT team,

- Other external agencies.

Of course, this doesn't exclude the need for direct financial support altogether, but the culture and approach described in this paper is at the heart of why the WUSAT Programme has been successful, and why it has become widely recognised within the Higher Education Space Engineering community.

Keywords-satellite engineering, WUSAT, project resourcing, funding, resourceful, inventive, attitude, culture, team-mentality, inclusivity, diversity.

\section{INTRODUCTION}

The University of Warwick Satellite Engineering Programme [1] is a series of Space-related activities based around the core activity of its Satellite Engineering Team. All activities work under the acronym, 'WUSAT'. The WUSAT project team is a multi-disciplinary team of $4^{\text {th }}$ Year MEng students. Their contribution to the current WUSAT project in any given year comprises $25 \%$ of their $4^{\text {th }}$ Year programme of study, and the School of Engineering provides a nominal amount of funding per student. However, the scale of WUSAT projects undertaken, and the high profile of WUSAT achievement, far outstrips the modest funding provided. Hence, measures to meet the shortfall are continually sought by the WUSAT Project Directors. At its inception in 2006, the WUSAT project team almost immediately became the primary electrical power supply subsystem team for a European Space Agency (ESA) Moon orbiting satellite (ESMO) [2].

Since 2012, WUSAT has developed its own CubeSats, each designed and built from first principles to meet a specific objective. This included a two-year project terminating in a successful sounding rocket launch (2013-15) via the ESA REXUS programme [3], and during 2015-to-Present, four years development of a 3U CubeSat designed for Low Earth Orbit via an ESA 'Fly Your Satellite via the ISS' programme [4]. The main objective of this latter project (WUSAT-3) is to provide a proof of concept for a RF signal direction finding technology that could enable the development of smaller, lighter wildlife tracking tags. This would enable a range of wildlife species, previously incapable of being monitored, to be tracked from LEO. It is clear that the scale of these projects requires a range of activities to be undertaken, and each of these can carry a substantial cost.

\section{Potential Costs}

For each WUSAT project these can include,

- Materials and components for prototyping, testing and eventual build

- Manufacturing costs

- Software licences and potential training for specific software packages 
- Travel and accommodation to attend ESA design review events

- The use of test facilities, including in-house facilities in some cases.

- Costs involved with developing and supporting company partnership links.

- The cost of producing promotional and outreach materials, and attending related events. This is considered a major priority for WUSAT as we promote our students, the School of Engineering, the University of Warwick, our partner companies, and ESA who themselves are providing launch opportunities via their outreach funding. This can take the form of team polo shirts (reproduced annually for each team) containing the logos of all of our partners, banners \& posters, travel/accommodation to give talks and attend conferences, etc.

- Potential costs incurred through registering radio communication frequencies for mission use.

- Insurance costs.

If admission to an ESA launch programme is obtained, an amount of ESA sponsorship will be offered in addition to the launch costs. However, this is often limited to a small number of team members attending an ESA programme event. Any other team members attending these events will require flight/accommodation costs, etc, to be sourced from the project budget.

In addition, due to the activities of WUSAT projects, the Directors of WUSAT are regularly invited to take part in a wider range of Space-related activities. These form the wider WUSAT Programme, and include;

- The formation of a 'virtual' research group of academics who have research specialisms potentially applicable to Space [5].

- Membership of a Midlands Innovation Space Group associated with the development of the new 'Space Park - Leicester' and looking at ways of developing a strong Midlands Space cluster of active universities and companies.

- A group formed through the Satellite Catapult to investigate the possible use of satellite technology to counter the activities of illegal wildlife poaching.

- Acting as the catalyst in a general review at the University of Warwick, designed to identify and bring together all potential resources that could combine to make Warwick a leading university in the area of Space Engineering and Technology.

We consider these activities to be an important part of WUSAT development. They increase our knowledge, extend our network of contacts and help to increase the profile of WUSAT, our partners, and the University of Warwick in general. However, they also have a cost in terms of time, effort and travel on the part of the two WUSAT Directors. This cost can be either sourced from the (already inadequate) WUSAT budget, or self-funded as a 'personal interest' activity.

Of course, the act of pursuing additional funding, in terms of determining what suitable funding is available and making an application for it, can itself be a costly exercise. Both WUSAT Directors are already highly committed/? in terms of the time given to both the WUSAT project and the wider WUSAT Programme, so finding the resource to pursue additional funding can itself be problematic. The remainder of this paper highlights the approach taken by the WUSAT Directors, and gives specific examples of measures employed in order to gain benefits for WUSAT that have allowed us to achieve a great deal on a minimum budget.

\section{CReating A TEAm CUlture fOR Everyone}

The main starting point when creating a working cultural environment that will encourage others to 'buy in' to your project, and eventually support you with it, is to provide INCLUSIVITY for everyone. In a university-based multidisciplinary engineering team, it is vital that a strong team ethic is in place in order for everyone to feel that they are fully playing their part whatever their particular skill or contribution.

However, it is just as important that other members of staff (technicians, academics, office staff, and university management) all feel that they are part of the extended 'team'. You will need things from all of these people over the lifetime of any significant Space project, so it is important to keep them in touch with progress, show your appreciation of what they do, and look for any opportunities whereby you can do something for them.

Similarly, when hoping to form partnerships with companies or other organisations, keep mentally putting yourself in their place. Rather than just thinking what you want from them, think what it is that they want from such a relationship with you. Companies rarely have access to a budget where they can just allocate funds to an educational project, but they will often have a wide range of other things they can offer you, and in many cases these can be worth a great deal of money if you had to fund them yourself (see later specific examples). Once you are able to talk with them, listen carefully to what they say. If you are inventive with your thoughts, you may spot opportunities where you can do something for them. Keep them informed of what you are doing and take every opportunity to include their name and contribution in your own publicity output.

When you nurture such relationships, it can sometimes be 'further down the road' before an opportunity suddenly arises whereby a relatively casual relationship with a company becomes very 'centre stage'. A need suddenly arises that has great interest and relevance to you and the company, and you are then pleased that you kept in touch and kept them informed!

An additional, and important, aspect of our 'team' approach at WUSAT has been to form strong links with the Warwick Aerospace Society. This has allowed us to run additional projects, e.g. CanSat, with teams formed by students from across a range of Warwick departments. This can have many benefits for WUSAT-3 and for our partners. 


\section{INITIATIVE - TYPICAL EXAMPLES}

This section is effectively the 'methodology' of this paper. It describes a range of initiatives that have not only increased the level of inclusivity in what we do, but has often brought considerable, direct benefits to our work. When operating on a minimal budget, these actions and the response they bring are often the difference between success and failure!

In most cases, except where it is unavoidable, we have kept the names of companies and individuals anonymous.

\section{A. WUSAT Team Day}

Towards the end of each academic year, we always hold a 'WUSAT Team Day'. We invite all of our participating partners to attend. The WUSAT Directors give a presentation on developments with the wider WUSAT programme, and the student team present their technical work and activities completed over the year. We then have a meal in a Warwick Conferences restaurant. There is ample time for networking, discussing new ideas and future plans. This is a most enjoyable day, and partner representatives really appreciate meeting the team and each other!

- Points to note - we do not refer to the companies as 'sponsors' as that implies a one-way relationship. We refer to them as 'partners', and the whole day as a 'Team Day' because we are all 'in the team', and that is the culture we wish to promote.

- Benefits -

- Company partners feel fully included as part of the team and want to contribute in any way they can because it is 'their' project and not just something they are supporting.

- Partners feel appreciated for what they do rather than taken for granted. Hence, they are often likely to offer more if they can.

\section{B. University Appointments}

A previous partner company representative wanted to remain research active to pursue information relative to the project. A letter written to the appropriate department at the University of Warwick to request that this person is appointed a Fellow of the University was successful. This gave them a Warwick University log-on, and access to research papers through the university library.

- Benefits -

- It gave the company employee access to a resource they could not easily acquire, and enabled them to research information relative to the project.

- It demonstrated that company/university relationships do not have to be 'one-way' but that WUSAT can offer benefits to the company too.

\section{Assisting University Staff}

Technicians in the University of Warwick engineering workshops are very skilled and do an excellent job. However, they are often under a lot of pressure to complete work for research and other student projects. Following the launch of WUSAT-2 in 2015, we learned that a submission was being made on their behalf for the National Papin HE Technician awards. We wrote a substantial reference in support of this submission, based on the excellent contribution they had made to the success of WUSAT-2. As a result, they were invited to attend the prestigious Awards Evening where they were awarded one of the top national prizes.

- Benefits -

- The engineering technicians have never forgotten that the WUSAT Team went 'out of its way' to support them. When there is pressure to prioritise work, WUSAT requests now always receive favourable consideration.

- The technicians now feel that they are part of the team, as indeed they are. We often invite them to presentations, etc, for that reason.

\section{Company Internships}

Helping to provide a good intern student to a company with a specific short-term need, can be a powerful way of assisting the company and potentially gaining favour with them if they can help you. For example - On meeting the CEO and representatives of a particular high-tech company, initial discussions seemed not much more than an exchange of information on our respective areas of work and the fact that 'somewhere down the line' there may be something we could do together. However, when the CEO happened to mention that they were mainly physicists who now needed to gain more engineering knowledge - particularly in the area of thermal modelling - we saw an immediate opportunity to provide them with a good mechanical engineering intern who could do just that. The company were delighted with this possibility, and when we managed to get the student appointed through the Warwick Summer Internship scheme, the student's six-week internship was also fully funded.

- Benefits -

- We earned the trust of the company because we made a suggestion and followed up on it for their benefit.

- We now have a close relationship with the company.

- They have generously offered us an expensive, flight-tested piece of equipment that we can use as part of the payload for WUSAT-3, plus a second that we can use as an engineering model for prototyping, etc. This would have cost us thousands of pounds if we had to procure it 
ourselves. We hope that both parties will now benefit from a very positive relationship.

Linking partner companies to any university internship scheme can be a very productive way of providing a benefit to the company and enhancing your relationship with them.

- $\quad$ Benefits -

- One beneficial outcome that companies often desire when supporting a university project is to recruit good quality graduates. Students completing an internship with a company is often the best way of the company seeing them at work and potentially pre-recruiting them prior to graduation.

- If a student is a potential recruit to your university team, arranging for them to do an internship with a partner company can be an ideal way of inculcating them into your project and your specific technical links to the company.

- We have had a number of occasions where a student on an internship with a non-partner company, then progresses into the WUSAT team. Due to their contacts and close ties with the company, they have then subsequently brokered a WUSAT partnership arrangement with them. This has been enormously beneficial for WUSAT in the past. Some of our major company partnerships have been obtained through that route, where otherwise 'cold calling' can be far less productive.

\section{E. Contributing to Company Promotional Activities}

Partner companies are very keen to promote their brand and to show their products/services being used in high-profile Space projects. Making the time and effort to help facilitate this with company partners can bring many rewards. WUSAT examples include;

1) Writing articles for a company newsletter, journal, or website.

2) Contributing to a company promotional video

3) Attending company outreach displays at, e.g. Science Fairs

4) Producing a case study for a company, often for display on their website.

5) Giving a presentation at a company/partner symposium

- $\quad$ Benefits -

○ (1) - Articles in company promotional outlets are also a 'free' promotion of your project and gives added kudos with your name linked to the company and their brand [6].

- (2) - WUSAT recently offered to support RS in a promotional video they were making. The result was also a brilliant promotional video for WUSAT itself [7]. In addition, during filming for the video, we happened to mention WUSAT's role in the forthcoming 2019 British Science Festival based at Warwick. RS responded by offering the use of their Titan demonstration lorry packed with interactive modern engineering technology [8]. So the outcome of our willingness to support RS was two significant contributions to WUSAT that we could not possibly have afforded.

○ (3) - Partner organisations will often attend outreach events in their own right, but will be keen to show their links to top-class university projects by having the project team and artefacts from the project on display alongside the organisation's main display. Again, this provides 'free' publicity for your own project, and enhances your standing with the partner company.

- (4) - WUSAT once collaborated in a major case study [9] with a software company, displayed on their website. The benefit was free licences for use of the design software by team members.

○ (5) - Partner organisations will often hold workshops/symposiums of their own [10], and you will enhance your standing with them, and will be much more likely to receive benefit from them, if you are willing to support these and make presentations, etc, when you can.

- $\quad$ Point to note -

The activities described above can be demanding and time-consuming when you already have a busy project to run. However, it is worth remembering that

a. If you receive funding from your university to run a high profile project, there is some expectation that you will promote it and engage in outreach activity, e.g. Open Days.

b. The partner organisations that are supporting you and lending their name to your project reasonably expect to see this promoted where possible.

c. If your project operates through, e.g. an ESA programme, then the funding and support received from them is part of their outreach funding. Hence, there is reasonable expectation that you will have some sort of outreach programme yourself. 
Hence, you will have some level of duty to take part in these activities in any case, so you may as well do it in collaboration with your partners and obtain the benefits that go with it.

\section{CONCLUSIONS}

Finding the time to produce additional promotional/outreach material, or pursue some initiative, for partner organisations can be difficult when you are running a busy project. It can be the easiest thing to ignore or put off for another time. However, the examples given in this paper show that the benefits derived from pursuing such activities when you can, can be incalculable.

If you are working on a limited budget and need additional resources to help you complete your project successfully, then adopting and developing this cultural way of working from the outset can reap enormous benefits. Of course, you must be careful not to say 'yes' to everything, but overall it is an extremely pleasant and rewarding way to expand the scope of your project and form many useful, close, working relationships.

Points to note -

- Student members of your team can of course, contribute to such activities. However, our experience is that even the most mature of students do not always have the confidence or experience to 'pitch' a particular approach to a potential partner in a way that makes it attractive to them. They are also not always aware of the range of benefits that may be available through university resources, etc. Hence, it is normally best for project supervisors/directors to lead on these matters. In any case, you normally want student team members to be working on technical matters!

- The limitation to this approach is, of course, flexibility. You can only obtain a desired product or service from an organisation if firstly, you are able to form a relationship with them and secondly, they are willing/able to make that provision because of your relationship with them. Clearly, you cannot just make a partner of every company that you need to acquire something from on the expectation that they will just give it to you. Most partnerships have to be nurtured on the sole expectation that they will offer you things when/if they can. Financial resources are still very much required.

- Any shortfall in funding, not forthcoming from sponsorship or benefits in kind, will be dependant upon the University underwriting the project. In order to achieve this commitment, the University needs to perceive that there is adequate benefit. The approaches identified above are fundamental to achieving the maximum benefit not only for WUSAT but also for the
University. Our focus on missions that have a realworld application has allowed us to enhance our outreach activities, with WUSAT-3's mission of wildlife monitoring providing an excellent opportunity to engage non-traditional audiences (e.g. gardening groups, bee-keepers, ecologists etc) in the excitement of an engineering project.

\section{ACKNOWLEDGMENTS}

We would like to acknowledge our extreme gratitude to the many partner organisations who have contributed to WUSAT over the past thirteen years. In no particular order - Harwin, RS Components, Roke Manor Research, Airbus, XCAM, Marks \& Clerk, AMSAT-UK, EuroCircuits, ESATAN TMS, Lotek (Biotrack), AGI, The Andrew Little Fund, Thales Alenia Space, Thales, GE Aviation, SSTL, National Instruments, Proctor Group, Prodrive, Qioptic, Plexim.

\section{REFERENCES}

[1] W. Crofts and J. Hunter-Anderson, "WUSAT - University of Warwick Satellite Engineering," [Online]. Available: www.warwick.ac.uk/cubesat. [Accessed 11 August 2019].

[2] European Space Agency, "ESMO Mission," [Online]. Available: http://www.esa.int/Education/ESMO_mission. [Accessed 12 August 2019].

[3] European Space Agency, "REXUS 17," [Online]. Available: http://www.esa.int/Education/University_student_experiments_take_to the_skies. [Accessed 12 August 2019].

[4] W. Crofts and J. Hunter-Anderson, "WUSAT-3 CubeSat Project," [Online]. https://warwick.ac.uk/fac/sci/eng/meng/wusat/projects/wusat-3/. [Accessed 13 August 2019].

[5] W. Crofts and J. Hunter-Anderson, "WUSAT Research," [Online]. Available: https://warwick.ac.uk/fac/sci/eng/meng/wusat/research/. [Accessed 13 August 2019].

[6] Harwin, "Monitoring Wildlife from Space Using CubeSats," [Online]. Available: https://www.harwin.com/blog/monitoring-wildlife-fromspace-using-cubesats/. [Accessed 13 August 2019].

[7] RS Components, "WUSAT- Engineers of Tomorrow - RS Components," [Online]. Available: https://uk.rsonline.com/web/generalDisplay.html?id=fortheinspired/warwickuniversity-satellite-team. [Accessed 13 August 2019].

[8] RS Components; Electrocomponents PLC, "Titan II: A Mobile Innovation Experience," [Online]. Available: https://www.electrocomponents.com/corporate-responsibility/ourcommunity/titan-ii. [Accessed 13 August 2019].

[9] Solid Solutions, "University of Warwick ESMO Project," [Online]. Available: https://www.solidsolutions.co.uk/solidworksIndustries/Case-Studies/University-of-Warwick.aspx. [Accessed 13 August 2019].

[10] AMSAT-UK, “2019 AMSAT-UK International Colloquium,”[Online]. Available: https://amsat-uk.org/colloquium/. [Accessed 13 August 2019]. 